\title{
FLOW FEATURES IN CHANNELS WITH PULSATING FLUID MOVEMENT REGIME
}

\author{
Natalia Sidenko, Egils Dzelzitis \\ Riga Technical University, Latvia \\ nsidenko@inbox.lv, egils.dzelzitis@rtu.lv
}

\begin{abstract}
Obtaining information about pulsating turbulent flow structure and identifying the heat transfer processes laws of such flows in the channels today is an urgent task. Identification of the physical causes of various factors that affect the flow structure and heat transfer in the channels can allow controlling of these processes. The numerical solution of the task is performed in the Flow Simulation software, in which the movement and heat transfer of fluids are modelled by using the Navier-Stokes equations. In this work is used a model of a periodically pulsating fluid flow with sinusoidal velocity pulses. The considered frequency range of the flow pulsations in the channel is from 0 up to $100 \mathrm{~Hz}$. The obtained calculation results with the flow pattern visualization make it possible to identify the flow features at given pulsations in the channel with respect to the stationary regime. The results of the numerical solution showed that flow pulsations significantly affect the heat transfer in the channels. At a flow pulsation frequency in the channel $0<f<40$, in comparing with the stationary mode, the Nusselt criterion increases by 10-15\%. At a frequency of $40<f<100$, the Nusselt criterion decreases by $10-15 \%$. The results of visualization of flow patterns in a discretely rough channel showed that the presence of vortex zones and zones of return flows leads to the destruction of the boundary layer and, as a consequence, to intensification of the heat transfer process.
\end{abstract}

Keywords: pulsating flows, Navier-Stokes equations, heat transfer, vortex zone, intensification.

\section{Introduction}

Much attention has been paid to the problem of studying the effect of pulsating flows on hydrodynamics and heat transferring in recent years. Experimental studies of unsteady flows at the present day showed that such unsteady process can be accompanied by both reduction and increasing in the intensity of heat exchanging [1].

Known results of experimental and theoretical [2-4] studies are often solutions for the specific problems and do not allow making broad generalizations about the level of influence of superimposed flow pulsations on the intensity of heat transferring. At this moment, there is not enough reliable information about the kinematic structure of the pulsating flow in the channels under the conditions of superimposed nonstationarity.

Based on the reliability of information about the structure of the pulsating flow in the channel, it is possible to estimate the effect of superimposed pulsations on the heat transferring, as well as to identify patterns of changing in the flow characteristics under considered conditions [5].

It is also known that increasing in the intensity of heat exchanging between the coolant and the heat exchange surface is provided, as a rule, with the help of discrete roughness, leading to partial or complete destruction of the boundary layer, in which the greatest thermal resistance is concentrated, as well as significant tubulisation of the flow. These effects are achieved by organization of flow separation and attachment areas, an increase in the relative flow velocity near the wall, and generation of the pressure pulsations in the flow and other ways of influencing the near-wall structure of the flow [6-8].

The experimental and theoretical results of the study of pulsating flows that exist today are mainly obtained only for the boundary layer and the flow in a smooth channel $[9 ; 10]$.

At this stage, numerical research methods using the latest advances in the field of the boundary layer diagnostics and computerization are the most effective way in the modern theory of heat and mass transferring.

The aim of this work is to study the effect of periodic flow pulsations on the causes and characteristics of heat transfer processes in a channel with discrete roughness in the shape of hemispherical concavities with using computer simulation and numerical analysis methods.

\section{Materials and methods}

Numerical studies were performed by using the CFD FlowSimulation computational aerohydrodynamics software. A three-dimensional model of a circular channel is considered, along the 
length of which hemispherical holes were installed in the corridor order. The geometrical dimensions are indicated in Table 1.

Table 1

\section{Channel geometrical dimensions}

\begin{tabular}{|c|c|c|}
\hline Name & Designation & Value \\
\hline Inner radius, $\mathrm{m}$ & $R$ & 0.009 \\
\hline Wall thickness, $\mathrm{m}$ & $\delta$ & 0.002 \\
\hline Length, $\mathrm{m}$ & $L$ & 1.6 \\
\hline Concavities spacing & $S \cdot h^{-1}$ & 10 \\
\hline Center angle, grad & $\varphi$ & 120 \\
\hline
\end{tabular}

To the solid walls of the channel were set properties of aluminum. The physical properties of the material at $T=378.15 \mathrm{~K}$ are shown in Table 2 .

Physical properties of aluminum

\begin{tabular}{|c|c|c|}
\hline Name & Designation & Value \\
\hline Density, $\mathrm{kg} \cdot \mathrm{m}^{-3}$ & $\rho$ & 2688.9 \\
\hline Specific heat, $\mathrm{J} \cdot(\mathrm{kg} \cdot \mathrm{K})^{-1}$ & $C p$ & $902-951$ \\
\hline Thermal conductivity, $\mathrm{W} \cdot(\mathrm{m} \cdot \mathrm{K})^{-1}$ & $q$ & $230-240$ \\
\hline Resistivity, Ohm $\cdot \mathrm{m}$ & $R$ & $4.29-5.97 \mathrm{e}-08$ \\
\hline Melting temperature, $\mathrm{K}$ & $T$ & 933.4 \\
\hline
\end{tabular}

Water as a working fluid was supplied to the channel. The physical properties of the liquid at a temperature of $293.15 \mathrm{~K}$ are shown in Table 3.

\section{Physical properties of the liquid}

Table 3

\begin{tabular}{|c|c|c|}
\hline Name & Designation & Value \\
\hline Density, $\mathrm{kg} \cdot \mathrm{m}^{-3}$ & $\rho$ & 998.6 \\
\hline Dynamic viscosity, $\mathrm{Pa} \cdot \mathrm{s}$ & $\mu$ & 0.0010014 \\
\hline Specific heat, $\mathrm{J} \cdot \mathrm{kg} \cdot \mathrm{K})^{-1}$ & $C p$ & 4184.4 \\
\hline${\text { Thermal conductivity, } \mathrm{W} \cdot(\mathrm{m} \cdot \mathrm{K})^{-1}}^{-1}$ & $q$ & 0.59843 \\
\hline
\end{tabular}

The set boundary conditions for the stationary case of solving the problem are summarized in Table 4 . Where $V_{\text {inlet }}$ - fluid (water) velocity at the channel inlet, $P_{\text {outlet }}$ - pressure at the outlet of the channel, $T_{\text {wall }}$ - temperature of the solid wall, $T_{\text {water }}$ - water temperature. Here is the Reynolds number for a given flow rate $V_{\text {inlet }}$ of fluid in the channel

$$
\operatorname{Re}_{0}=\frac{V_{\text {inlet }} \cdot D \cdot \rho}{\mu} .
$$

\section{Boundary conditions}

Table 4

\begin{tabular}{|c|c|c|c|c|}
\hline $\boldsymbol{V}_{\text {inlet }}, \mathbf{m} \cdot \mathbf{s}^{-\mathbf{1}}$ & $\boldsymbol{R e}_{\mathbf{0}}$ & $\boldsymbol{P}_{\text {outlet, }} \mathbf{P a}$ & $\boldsymbol{T}_{\text {wall }}, \mathbf{K}$ & $\boldsymbol{T}_{\text {water, }}, \mathbf{K}$ \\
\hline 0.3 & 5384 & - & - & - \\
\hline 0.4 & 7179 & - & - & - \\
\hline 0.5 & 8974 & - & - & - \\
\hline 0.6 & 10769 & 10325 & 378.15 & 293.15 \\
\hline 0.7 & 12564 & - & - & - \\
\hline 0.8 & 14359 & - & - & - \\
\hline 0.9 & 16154 & - & - & - \\
\hline 1 & 17949 & - & - & - \\
\hline
\end{tabular}

Diagram of the boundary conditions is shown in Fig. 1. 

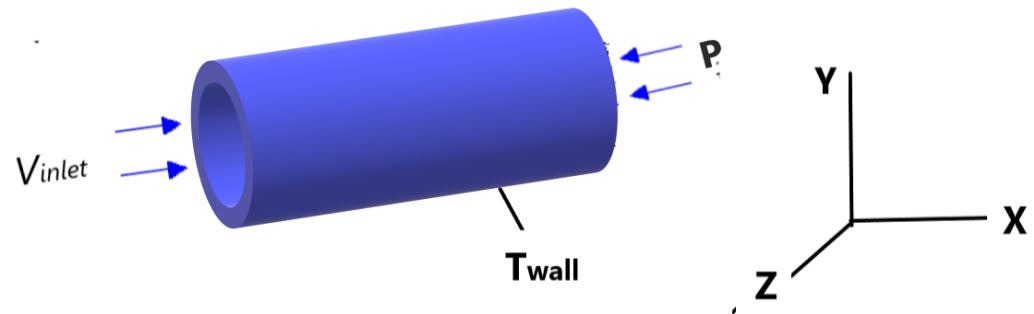

Fig. 1. Diagram of the boundary conditions in the channel

For the case of periodic pulsations of the fluid flow in the channel, the $R e_{t}$ number depends on time and is described by equation (1):

$$
R e_{t}=\left(1+A \sin (2 \cdot \pi \cdot f \cdot t) \cdot \frac{V_{\text {inlet }} \cdot D \cdot \rho}{\mu},\right.
$$

Where $t$-time, s;

$A$ - amplitude of the working fluid pulsations, $A=1$;

$f$ - frequency of the working fluid flow pulsations, $\mathrm{Hz}$.

The motion and heat transfer of a fluid is modeled by using of the Navier - Stokes equations, which describe the laws of conservation of mass, momentum, and energy in a non-stationary formulation. Also, when solving the problem, the equations of state of the components of the fluid, and the empirical dependence of the viscosity and thermal conductivity of the medium on temperature are used [11-13].

In all studied cases of numerical solution of the problem, the flow is considered as turbulent. That is, the Navier-Stokes equations are averaged according to Reynolds, as a result, they have additional terms - Reynolds stresses. To close this system of equations, a $k-\varepsilon$ turbulence model is used.

The obtained numerical values according to the results of computer calculations were processed according to well-known methods of the theory of similarity of processing the hydrothermodynamic experiment [11-13].

\section{Results and discussion}

Calculation results for the heat exchange in smooth channels are well understood, unlike the rough discrete channels. Therefore, the first stage of the problem under consideration was the solution of the test problems in a smooth channel and the estimation of the accuracy of the obtained solutions. Numerical solutions in a smooth channel: channel geometry, radius, wall thickness and channel length are shown in Table 1. Physical properties of the material and liquid are given in Table 2, Table 3. Boundary conditions - Table 4.

Comparison of the results of numerical calculations with theoretical ones in a smooth channel for the heat transfer coefficient $N u_{\text {theory }}$ (theoretical calculation, according to [14]) and $N u_{\text {smooth }}$ (numerical solution), for initial Reynolds numbers $R e_{0}=5000-18000$, showed an acceptable accuracy in the range of 5-10\%. Summarizing of the results of solving problems is shown in Table 5.

At the next stage of the study, the problem was considered in a channel with discrete roughness, under the same conditions specified in Tables 1-4. The results of numerical calculations showed that in comparison with a smooth channel, the Nuselt number $\left(N u_{\text {theory }}, N u_{s m o o t h}\right)$ in a discrete rough channel $\left(N u_{\text {discrete }}\right)$ - increases by 2 times (Table 5 ).

Here, all considered parameters were calculated at given points at the channel exit $[12 ; 13]$. The data obtained are confirmed by some well-known studies [14].

The second stage included numerical studies for the case of a periodically pulsating fluid flow, where the Reynolds criterion is described by equation (1). The geometry of the channels, the physical properties of the material and liquid were similar, as in the first problem. A distinctive feature for studying the influence of flow pulsations on the heat transfer in the channels was that, under boundary conditions, a sinusoidal pulse was supplied at the channel inlet with a frequency of the fluid flow pulsations $0<f<100 \mathrm{~Hz}$, for $V_{\text {inlet }}=0.4 \mathrm{~m} \cdot \mathrm{s}^{-1}$ and $R e_{0}=7179$. 


\section{Comparative table of the obtained solutions results for the Nusselt number}

Table 5

\begin{tabular}{|c|c|c|c|c|}
\hline $\boldsymbol{V}_{\text {inlet }} \mathbf{m} \cdot \mathbf{s}^{-\mathbf{1}}$ & $\boldsymbol{R e}_{\mathbf{0}}$ & $\boldsymbol{N u}_{\text {theory }}$ & $\boldsymbol{N \boldsymbol { u } _ { \text { smooth } }}$ & $\boldsymbol{N} \boldsymbol{u}_{\text {discrete }}$ \\
\hline 0.3 & 5384 & 66 & 67 & 149 \\
\hline 0.4 & 7179 & 81 & 84 & 187 \\
\hline 0.5 & 8974 & 88 & 101 & 222 \\
\hline 0.6 & 10769 & 115 & 116 & 256 \\
\hline 0.7 & 12564 & 130 & 132 & 290 \\
\hline 0.8 & 14359 & 147 & 147 & 323 \\
\hline 0.9 & 16154 & 160 & 161 & 361 \\
\hline 1 & 17949 & 172 & 175 & 390 \\
\hline
\end{tabular}

The results of numerical studies showed that, in contrast to the stationary case of the flow, in the considering channels a periodic change in time of the Re criterion (according to equation (1) significantly affects the $N u$ number. And the consequence leads to its change in time.

Fig. 2 presents the results of the studied parameter of the $N u$ number calculated over the entire calculated region of the channel, and shows the dependence of the Nusselt number $(\mathrm{Nu})$ on time $(t)$, at a flow pulsation frequency $f=20 \mathrm{~Hz}$, for a smooth channel $-\mathrm{a}$, and discrete rough $-\mathrm{b}$. Such effect is due to the periodic change of the Reynolds number and the inertia of the processes under unsteady fluid flow in the channels.
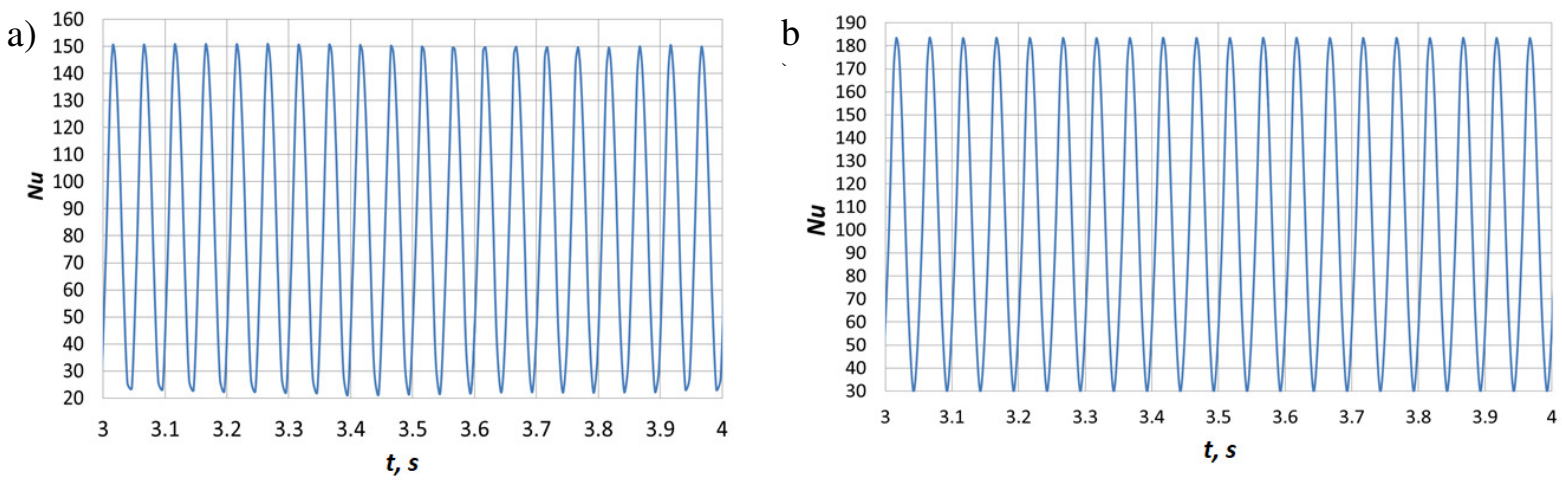

Fig. 2. Dependence of the Nusselt number $N u(t)$, at $f=20 \mathrm{~Hz}$ :

$a$ - smooth channel; $b$ - discrete rough channel

During the study of heat transfer in the outlet sections of the channels, it was found that periodic pulsations of the fluid flow can lead to both improvement and deterioration of the average values of the Nuselt criterion depending on the flow frequency pulsations. For both cases, a smooth and discretely rough channel, with a pulsation frequency of $0<f<40$, the average value increases and with $40<f<100$ it decreases. In both cases, the value in relation to the average value reaches 10$15 \%$.

The third stage covered visualization of the channel flow in the concavity area, in order to identify features that affect heat transfer.

Fig. 3 shows visualization of a flow in a channel with discrete roughness (longitudinal section) in the case of stationary flow of a fluid at average speed at the entrance to the channel $V_{\text {inlet }}=0.4 \mathrm{~m} \cdot \mathrm{s}^{-1}$. The flow under consideration can be conditionally divided into three areas: I - the outer boundary layer, II - the mixing region, and III - the return flow.

The flow picture can be presented as follows. The incident flow $\mathrm{V}$ at the exit from the concavity is turbulized, mixed with the medium in the concavity, and carries particles of the liquid from the mixing zone to the main flow. At the same time at environ of the outlet edge the flow is splitting. One part of the flow (laminar sublayer) moves along the straight wall of the channel, the other part goes around the surface of the concavity, and when it reaches the input edge of the concavity (inlet edge), it breaks down forming a vortex. 


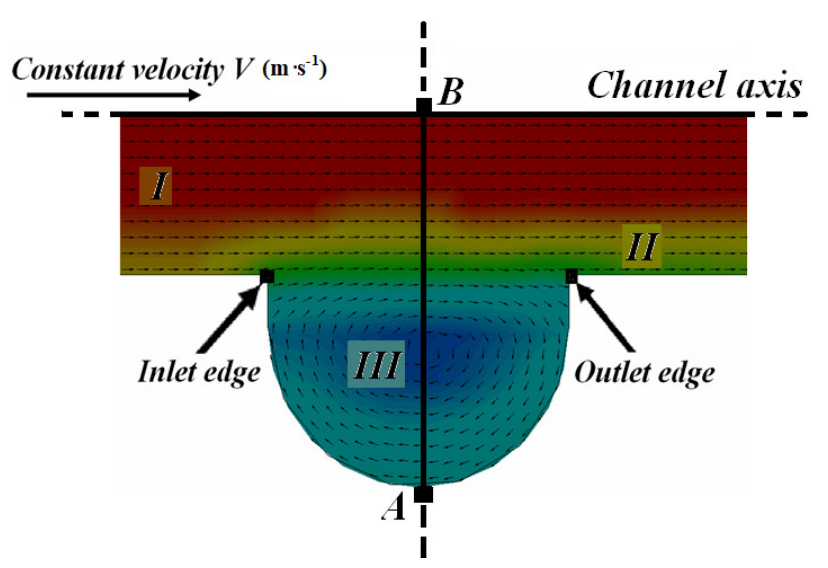

Fig. 3. Flow pattern with a stationary flow $V_{\text {inlet }}=0.4 \mathrm{~m} \cdot \mathrm{s}^{-1}$ concavities in the channel (longitudinal section)

Comparing with the stationary motion in the pulsating mode, the flow patterns are changing at time $t(s)$. Unlike the stationary flow, in the pulsating mode at an average flow velocity of $V_{\text {aver }}(t)=0.4 \mathrm{~m} \cdot \mathrm{s}^{-1}$ (equation (1)), the pulsating frequency $f=20 \mathrm{~Hz}(S h=0.9) R e \approx 27000 \mathrm{Fig}$. 4, the flow patterns are changing. Here, the inlet flow $V_{\text {aver }}$ moves along a straight channel, when the input edge of the concavity (inlet edge) is reached, the flow is splitting: one part of the flow moves towards the output edge (outlet edge), the other part towards the point 0 , where it is turbulized and mixed. So, unlike in the stationary mode, it shifts from the outlet edge (outlet edge) towards the axis $\mathrm{AB}$ by approximately $45^{\circ}$. In the same place, at point 0 , one more splitting of the flow occurs. One part of it (laminar sublayer) moves along the surface of the concavity towards the outlet edge (outlet edge) and then continues to move along the straight channel wall. The other part flows around the surface of the concavity, and when it reaches point $C$, it breaks down forming a vortex. At the same time, unlike the stationary flow, the center of the vortex that was on the axis $\mathrm{AB}$, in the pulsating mode, the center of the vortex shifts towards the input edge by a distance approximately equal to half of the radius of the concavity.

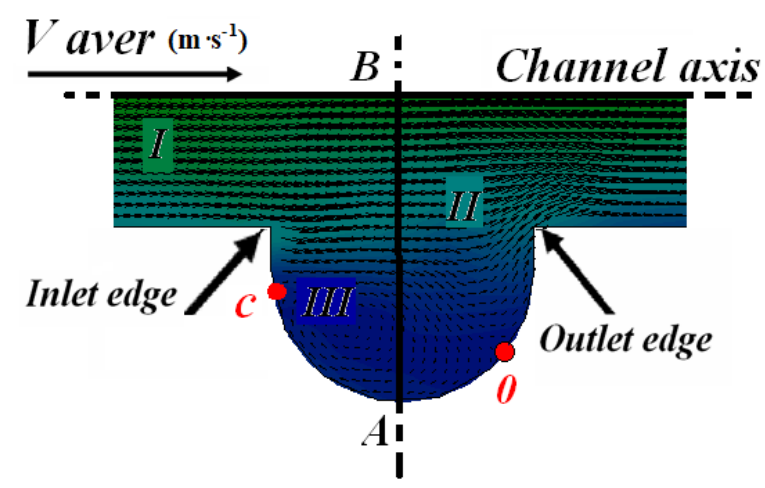

Fig. 4. Flow around the pulsating flow $V_{a v e r}(t) \mathrm{m} \cdot \mathrm{s}^{-1}$ concavity in the channel (longitudinal section) $f=20 \mathrm{~Hz}(S h=0.9)$

When the maximum flow rate $V_{\max }(t)$ is reached, the flow pattern is simplified, the flow moves along the straight channel wall, and when the input edge (inlet edge) is reached, part of the flow moves in a straight line to the output edge (outlet edge), and a part of flow around the concavity and joins the main flow around the exit edge. At the same time, no flow separation or vortex zones are observed in the concavity, Fig. 5.

The complex flow pattern is observed, when the minimum flow rate $V_{\min }(t)$ is reached, Fig. 6. Here the flow in the laminar sublayer changes its direction to the opposite in relation to the inlet flow and moves from the output edge (outlet edge) to the input edge (inlet edge). When reaching the entrance edge, the flow is splitting and part of it is moving along the straight wall of the channel, and part begins to move closer to the channel axis, where it merges with the main fluid flow and thus 
forms a vortex zone III. The centre of the vortex is located on the axis $\mathrm{AB}$ and at a distance approximately equal to half the radius of the smooth part of the channel.

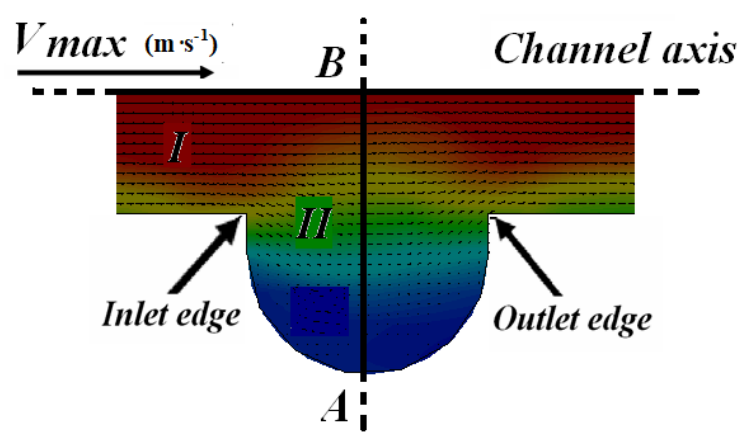

Fig. 5. Flow around the pulsating flow $V_{\max }(t), \mathrm{m} \cdot \mathrm{s}^{-1}$ concavity in the channel (longitudinal section) $f=20 \mathrm{~Hz}(S h=0.9)$

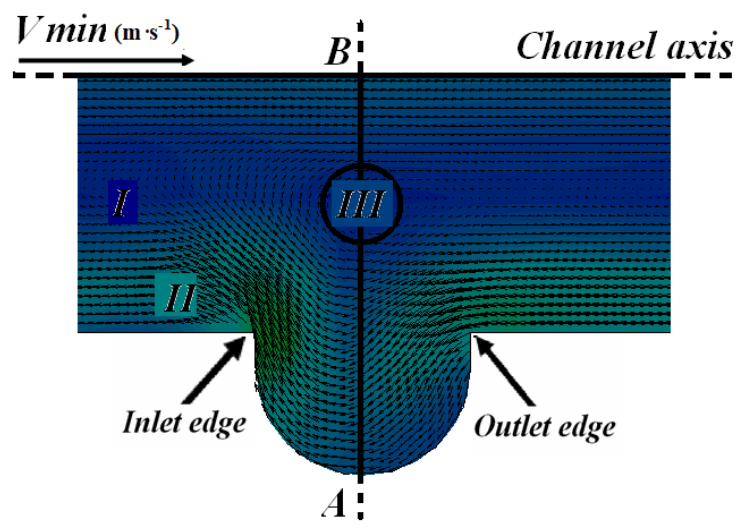

Fig. 6. Flow around the pulsating flow $V_{\min }(t), \mathrm{m} \cdot \mathrm{s}^{-1}$ concavity in the channel (longitudinal section) $f=20 \mathrm{~Hz}(S h=0.9)$

Changing in the Nusselt number in the cross section $\mathrm{AB}$ for the minimum, maximum and average values of the flow velocity at a flow pulsation frequency of 0 and $20 \mathrm{~Hz}$ is shown in Fig. 7.

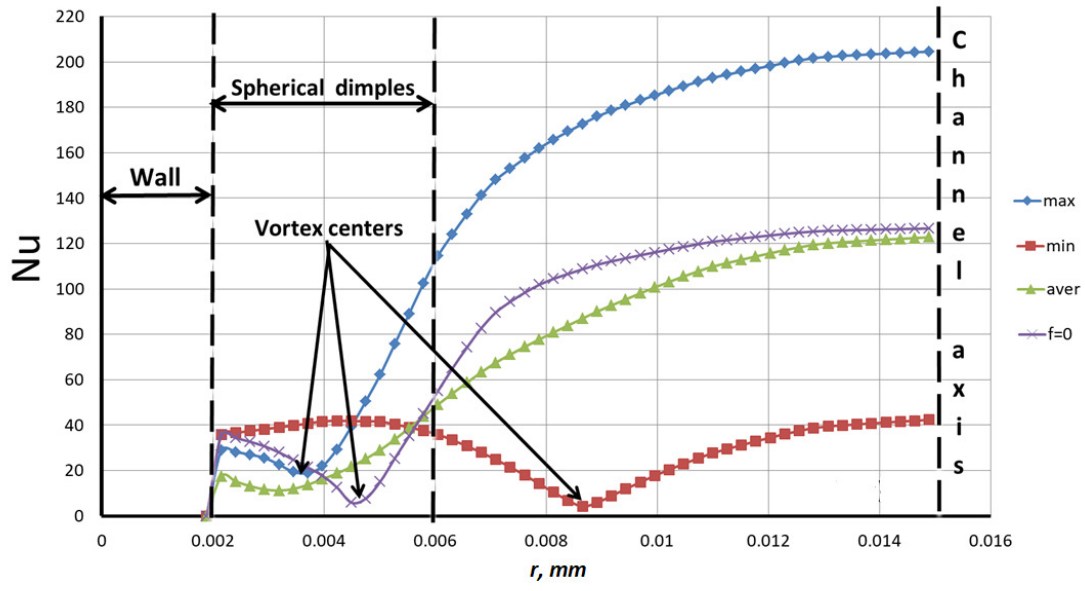

Fig. 7. Dependence of the Nusslet number along the radius of the channel in the $\mathrm{AB}$ cross section

So, it can be assumed that the presence of vortex structures and return flow zones on the heat exchanging surface leads to the destruction of the boundary layer and, accordingly, intensifies the heat exchange processes. In this case, the return flows that occur when the minimum flow rate is reached worsen the heat transfer process.

And the occurrence of such disturbing factors in the flow significantly affects both the hydrodynamics of the flow and the heat transferring. 


\section{Conclusions}

1. Due to the difficulty of solving the research task and the limited experimental results, the studies were carried out in several stages. At the first stage, simplified test problems were considered, which made it possible to evaluate the accuracy of the numerical solutions obtained by comparing them with known theoretical data. The results showed a satisfactory convergence of the calculations of $5-10 \%$.

2. The second stage involved the complication of the task based on the already obtained numerical solutions. The case of a periodically pulsating fluid flow in the channels was considered. The results of the numerical solutions showed that flow pulsations significantly affect the heat transfer in the channels. The pulsation frequency of the flow $0<f<40$ can lead to an improvement in the Nusselt criterion by $10-15 \%$. And at $40<f<100$ it can lead to its deterioration also by $10-15 \%$.

3. At the third stage of the study, when visualizing the flow in the channels, the reasons were established that affect the heat transfer in a discrete rough channel in the presence of the fluid flow pulsations. Comparing with the stationary motion in a pulsating mode in a discrete rough channel, the flow patterns change at time t. It was revealed that for the average flow velocities in the cavity region the flow is separating and deviation of the vortex zone is observed in the cavity region at a distance of approximately half of the cavity radius. For the maximum velocities, in the flow pattern there are no separated flows and, as a consequence, vortex zones. When the minimum speed is reached, forming return flows, at the entrance edge of the cavity the flow splits and the vortex zone shifts to the smooth part of the channel. These effects lead to destruction of the boundary layer and, as a consequence, to the intensification of the heat transfer processes.

\section{Acknowledgements}

This work has been supported by the European Regional Development Fund within the Activity 1.1.1.2 "Post-doctoral Research Aid" of the Specific Aid Objective 1.1.1 "To increase the research and innovative capacity of scientific institutions of Latvia and the ability to attract external financing, investing in human resources and infrastructure" of the Operational Programme "Growth and Employment" (No Nr.1.1.1.2./VIAA/1/16/093).

\section{References}

[1] Galicejskij B.M. Ryzhov YU.A., Yakush E.V. Thermal and hydrodynamic processes in oscillating flows. Int:Mechanical Engineering, 1977. 256 p.

[2] Kiselev N.A., Burtsev S.A., Strongin M.M. A procedure for determining the heat transfer coefficients of surfaces with regular relief. Measurement Techniques, 2015, vol. 58, no. 9, pp. 1016-1022. DOI: $10.1007 \cdot \mathrm{s}^{-1} 11018-015-0835-7$

[3] Leontiev A.I., Dilevskaya E.V., Vinogradov Yu.A., Yermolaev I.K., Strongin M.M., Bednov S.M., Golikov A.N. Effect of vortex flows at surface with hollow-type relief on heat transfer coefficients and equilibrium temperature in supersonic flow. Experimental Thermal and Fluid Science, 2002, vol. 26, iss. 5, pp. 487-497. DOI: $10.1016 \cdot \mathrm{s}^{-1} 0894-1777(02) 00157-7$

[4] Mizushina T., Maruyama T., Hirasava H. Strukture of the turbulence in pulsating pipe flows. Eng. Japan -1975-Vol.8, Nr.3-p.210-216.

[5] Davletshin I.A., Paereliy A.A. "Behavior of heattransfer in pulsating flow in the channel entrance region", Journal of Physics: Conference Series, 2017

[6] Chang P. Separation of Flow //New York, Pergamon Press, 1971.

[7] Kiselev N.A., Leontiev A.I., Vinogradov Yu.A., Zditovets A.G., Strongin M.M. "Effect of largescale vortex induced by a cylinder on the drag and heat transfer coefficients of smooth and dimpled surfaces", International Journal of Thermal Sciences, 2019

[8] Kiselev N.A., Burtsev S.A., Strongin M.M. "A Procedure for Determining the Heat Transfer Coefficients of Surfaces with Regular Relief", Measurement Techniques, 2015

[9] Saric S., Jakirlic S., Tropea C. A Periodically Perturbed Backward-Facing Step Flow by Means of LES, DES and T-RANS: An Example of Flow Separation Control // Journal of Fluids Engineering. Sept. 2005. Vol.127. pp. 879-887.

[10] Alyamovsky A.A. and others. SolidWorks 2007/2008. Computer modeling in engineering practice. // BHV- Petersburg, Saint-Petersburg, 2008. 
[11] Sidenko N., Dzelzitis E. The method of numerical modeling of hydrodynamics and heat exchange in a channel with discrete roughness. International Scientific Journal „Mathematical Modeling” 2018, pp. 21-25.

[12] Dzelzitis E., Sidenko N. Numerical simulation of heat exchange in discrete-rough channels at flow superimposed oscillations. Engineering for rural development. Jelgava, 22.-24.05.2019 pp. 1155-1161. DOI: 10.22616/ERDev2019.18.N203.

[13] Mikheev N.I. Heat exchange at the separation of a pulsating flow / N.I.Miheev, I.A. Davletshin, V.M. Molochnikov // Thermal processes in engineering. 2009. F.1. No.8. pp. 314-317. 